\title{
Search for Pulsed Emission in Archival VERITAS Data
}

\author{
Avery Archer* for The VERITAS Collaboration ${ }^{\dagger}$ \\ Washington University in St. Louis \\ E-mail: a.archerewustl.edu
}

\begin{abstract}
Since the 2011 VERITAS discovery of very high energy (VHE; E>100 GeV) gamma rays from the Crab pulsar, there has been concerted effort by the gamma-ray astrophysics community to detect other pulsars in the VHE band in order to place better constraints on emission models. Pulsar modelling demonstrates that much of the magnetosphere is opaque to VHE photons, limiting emission regions to the outer magnetosphere or beyond the light cylinder. The locations of 19 known pulsars have been observed by VERITAS since full observations began in 2007 with 11 locations having more than 20 hours of observations. Observations of VHE emission from more sources could provide key data to help constrain current models of emission location and mechanisms. We present the status of the ongoing VERITAS program searching for pulsed emission in archival data.
\end{abstract}

The 34th International Cosmic Ray Conference

30 July- 6 August, 2015

The Hague, The Netherlands

\footnotetext{
*Speaker

†'http://veritas.sao.arizona.edu
} 


\section{Introduction}

In 2011 the VERITAS and MAGIC collaborations announced the first discovery of pulsed emission from a pulsar, the Crab pulsar, in the VHE regime [1], [2]. Prior to these observations, 46 gamma ray pulsars were known to exist with 7 having been detected by instruments on board the CGRO [3],[4] and the remaining 39 newly discovered by the Fermi-LAT [5]". The spectra of these pulsars were thought to have an exponential cutoff bolstering the curvature radiation scenario which predicts a spectral cut-off of this shape [6]. The discovery of VHE gamma ray pulsations from the Crab pulsar has challenged current emission mechanism models. The curvature radiation scenario alone does not adequately explain the VHE emission. A combined spectral energy distribution (SED) from Fermi-LAT and VERITAS, over the energy range 100 $\mathrm{MeV}$ to several hundred $\mathrm{GeV}$, clearly favors a broken power law of the form $A \times$ $\left(E / E_{0}\right)^{\alpha} /\left[1+\left(E / E_{0}\right)^{\alpha-\beta}\right][1]$. Many alternative emission scenarios have been proposed to explain the VHE emission from the Crab pulsar, including inverse-Compton (IC) scenarios in the outer magnetosphere [7,8] or beyond the light cylinder [9]. The discovery of, or strong observational upper limits on, VHE emission from other pulsars will provide critical measurements of the shape of the emission spectrum above the apparent $\mathrm{GeV}$ break and thus strongly constrain plausible emission scenarios.

Since the discovery of pulsed emission from the Crab pulsar, there has been a concerted and ongoing effort by the gamma-ray astronomy community to add to the VHE pulsar catalogue and constrain emission models. The Vela pulsar has been detected by HESS [10] above $30 \mathrm{GeV}$ and by the Fermi-LAT above $50 \mathrm{GeV}$ [11]. VERITAS has recently reported observations of the Geminga pulsar, the second-brightest steady, high-energy $(\mathrm{E}>100 \mathrm{MeV})$ gamma-ray source in the sky. These observations yield no evidence for periodicity in the VHE band. Upper limits above $166 \mathrm{GeV}$ are reported here [12]. The Crab pulsar remains the only currently known VHE emitting pulsar. A recent stacked analysis of 115 Fermi-LAT pulsars found no significant cumulative excess above $50 \mathrm{GeV}$. The stacked analysis constrains the average flux of the 115 pulsars analyzed but does not exclude the possibility of finding pulsars which are as bright as the Crab pulsar above $50 \mathrm{GeV}[13]$.

The locations of 19 Fermi-LAT pulsars have been observed since full observations with VERITAS began in 2007. Each of these pulsars are new candidate VHE sources and most have not been observed by other VHE instruments. Many of these observations were obtained when the pulsar was in the field of view of another primary target. 11 of the locations have more than 20 hours of observations each. VERITAS has the capability of detecting a 1\% Crab Nebula flux from a point source in roughly 25 hours. The top 10 pulsars, in terms of spin-down power divided by distance squared, detected in the Northern Hemisphere by Fermi-LAT are contained in this archival dataset. In this paper we present the ongoing effort by VERITAS to search for pulsed emission from these known pulsars and add to the VHE pulsar catalog.

\section{Fermi-LAT Analysis}

A detailed Fermi-LAT analysis has been performed on each pulsar listed in Table 1 using 5.2 years of Fermi-LAT data to produce phase-resolved SEDs ${ }^{\S}$. The phase-resolved SEDs

\footnotetext{
$\$$ In the recent 3rd Fermi-LAT Source Catalog (3FGL) the number of known gamma ray pulsars has increased to $143[14]$

$\S$ The size of the data set used to produce the SEDs is determined by the period of validty of the pulsar timing solutions used.
} 
include relevant phase cuts in order to avoid contamination from un-pulsed emission (e.g. pulsar wind nebula). These SEDs (not included in this proceeding) provide a measurement of the spectral shape above the break energy to enable possible extrapolations of flux in the VHE band.

Presented in Figure 1. are the phase-averaged SEDs for 18 of these 19 pulsars as measured in the Fermi Large Area Telescope Third Source Catalog (3FGL) [15]. The procedure for creating the SEDs are described in the 3FGL. These SEDs have no phase-cut applied to exclude steady-state emission, thus the spectral shapes may shown may be a combination of pulsed and un-pulsed nebula emission.

For most pulsars, the spectral shape above the break is sufficiently well measured to preclude extrapolations which connect smoothly to the Fermi-LAT SED but predict a VHE flux above $1 \%$ of the Crab Nebula flux. Given this, VERITAS will adopt a broad search strategy, which includes investigations of VHE emission which manifests as a new spectral component, not connected to the Fermi-LAT SED. Such a ccomponent may arise due IC scattering. [16].

\begin{tabular}{c|c|c|c} 
Source & Exposure (min) & Average Elevation $(\mathbf{d e g})$ & Average Offset (deg) \\
\hline PSR J0007+7303 & 2125 & 47 & 0.8 \\
\hline PSR J0023+0923 & 924 & 63 & 0.65 \\
\hline PSR J0205+6449 & 529 & 55 & 0.46 \\
\hline PSR J0218+4232 & 2043 & 73 & 1.06 \\
\hline PSR J0248+6021 & 1706 & 58 & 0.96 \\
\hline PSR J0357+3205 & 4035 & 76 & 0.9 \\
\hline PSR J0631+1036 & 563 & 66 & 0.86 \\
\hline PSR J0633+0632 & 832 & 61 & 1.07 \\
\hline PSR J0659+1414 & 7663 & 66 & 1.04 \\
\hline PSR J1907+0602 & 585 & 60 & 0.95 \\
\hline PSR J1932+1916 & 3577 & 69 & 0.94 \\
\hline PSR J1939+2134 & 1101 & 74 & 0.94 \\
\hline PSR J1954+2836 & 457 & 75 & 0.96 \\
\hline PSR J2021+3651 & 5393 & 72 & 0.91 \\
\hline PSR J2021+4026 & 2069 & 67 & 0.91 \\
\hline PSR J2032+4127 & 3842 & 70 & 0.85 \\
\hline PSR J2229+6114 & 3884 & 57 & 0.87 \\
\hline PSR J2238+5903 & 480 & 69 & 0.87 \\
\hline PSR J2240+5832 & 340 & 69 & 0.88
\end{tabular}

Table 1: Summary of VERITAS observations. From left to right: source name, preliminary exposure time in minutes, average elevation in degrees, and preliminary average offset in degrees.

\section{VERITAS Observations and Analysis}

The Very Energetic Radiation Imaging Telescope Array System (VERITAS), located at the Fred Lawrence Whipple Observatory (FLWO) in southern Arizona (31 40N, 110 57W, $1.3 \mathrm{~km}$ a.s.1.) is an array of four 12 -meter diameter imaging atmospheric Cherenkov telescopes [17]. VERITAS is sensitive in the energy range of $85 \mathrm{GeV}$ to $>30 \mathrm{TeV}$. VERITAS has an energy resolution of $15 \%-25 \%$ at $1 \mathrm{TeV}$ and a typical angular resolution of $<0.1^{\circ}$.

As mentioned in the previous section, the locations of 19 known pulsars have been observed by VERITAS with 11 locations having 20 hours or more of observations. After eliminating data affected by technical problems or taken under variable or poor sky conditions the total archival data set of known pulsars comprises more than 700 hours of observations 
carried out under four-telescope operations. A list of the observed targets and details of observations taken for each pulsar can be found in Table 1 .

\subsection{Phase-Gate Selection Procedure}

The procedure for defining phase gates for pulsed emission searches in VERITAS data determines optimal phase gates to use for VHE pulsed analysis given an exposure time, an assumed VHE source flux and a phaseogram published in the supplementary material of the 2nd Fermi-LAT Pulsar Catalog (2PC) [14]. Event rates for signal and background regions are obtained from a reflected region analysis [18] of the Crab Nebula and event selection criteria that are optimal for sources with $1 \% \mathrm{Crab}$ Nebula flux. Specifically, the rates of ON events $\left(N_{O N}\right)$ and $\alpha$-scaled OFF $\left(\alpha N_{O F F}\right)$ events are obtained. The rates are then multiplied by the VERITAS exposure time on a given pulsar to obtain the $N_{O N}$ and $\alpha N_{O F F}$ events for the given exposure time. The number of excess events $\left(N_{\text {excess }}\right)$ is found by $N_{\text {excess }}=N_{O N}-\alpha N_{O F F} . N_{\text {excess }}$ is then scaled to $1 \%$ of the calculated value, corresponding to an assumed VHE source flux of $1 \%$ of the Crab nebula. The Fermi-LAT 2PC phasogram for the source is used, and the value of the least populated bin is subtracted from all of the bins in the phaseogram in order to subtract the un-pulsed background. The background subtracted phaseogram is then normalized, and each bin of the background subtracted normalized phaseogram is multiplied by the scaled excess. Background counts determined from the offline analysis of the Crab Nebula are then added. Specifically, the number of $\alpha$-scaled OFF counts divided by the number of bins in the phaseogram $\left(\alpha N_{O F F} / N_{b i n s}\right)$ is added to each bin of the phaseogram. All non-overlapping phase-gate combinations are tested with optional constraints on minimum and maximum phases and gate widths for each phase gate and number of signal phase gates. The number of events in the signal and background regions for a tested combination of gates are used to calculate a significance using equation (17) from [19]. The combination of gates that results in the highest significance is saved and plotted as shown in Figure 2. These phase gates will be used in the analysis of VERITAS archival data.

The number of signal phase gates for a given pulsar is chosen based on a visual inspection of the Fermi-LAT phaseogram. For 100 bin phaseograms, the maximum tested gate widths were constrained to be larger than the widest visually identified signal and background regions but smaller than the width of the entire phaseogram. For phaseograms with two peaks with different heights, phase gates are not defined for the small peaks using the procedure described above. A three pass procedure is used to define phase gates for the small peaks in these cases. The first pass consists of the method described above. The second pass defines only one signal phase gate, and excludes the gate defined for the large peak in the first pass from the signal gate search region. This ensures a gate is defined for the small peak. The third pass defines two signal phase gates but constrains one of the signal gates to be the one defined in the second pass. The three pass procedure effectively defines phase gates for the small peaks in these cases and was used to define the gates for PSRJ0205+6449 and PSRJ2021+4026. 

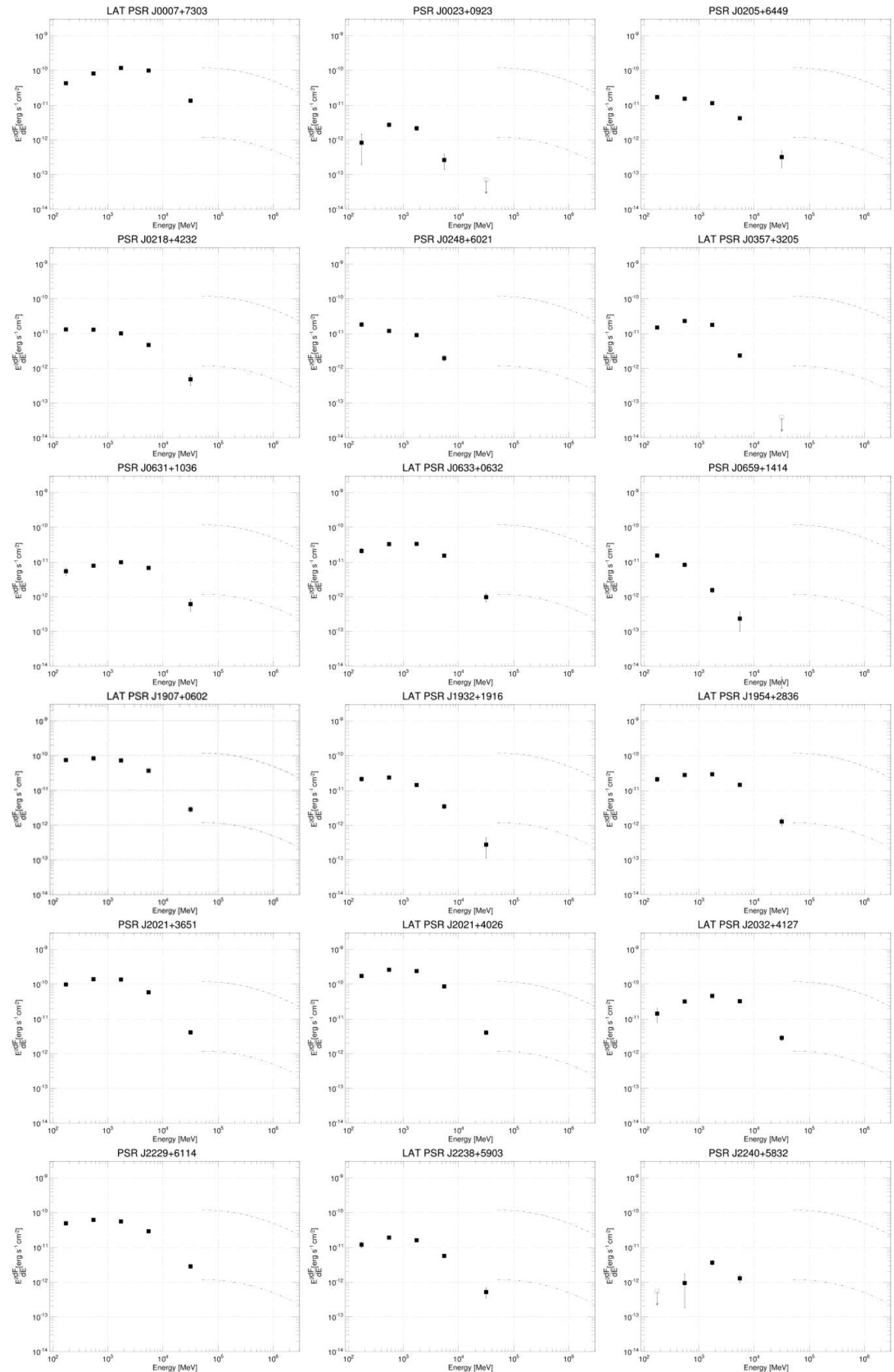

Figure 1: Phase-averaged SEDs from 3FGL for each of the 18 pulsars in VERITAS archival data (excluding PSRJ1939+2134). Measurements of 100\% Crab Nebula flux and 1\% Crab Nebula flux are plotted for comparison. 


\section{Discussion and Future Plans}

Pulsar studies with VERITAS are an active and ongoing effort. Last year the collaboration developed a Long Term Plan to map out scientific priorities for the next five years and pulsar studies are one priority in the Long Term Plan. The VERITAS pulsed analysis of the pulsars in the VERITAS archival data is not fully completed and results of these analyses are forthcoming.

In addition to analysis of archival data, different populations of pulsars are being examined for targeted observations. One class of pulsar that may be of particular interest are millisecond pulsars. With very short spin periods, the light cylinders of these pulsars have smaller radii and thus more compact magnetospheres than other pulsars. Observations of millisecond pulsars can serve as another avenue to constrain VHE emission models and mechanisms. For more details on the constraints that millisecond pulsars can provide see the VERITAS contriubtion to this conference [20].

\section{Acknowledgements}

This research is supported by grants from the U.S. Department of Energy Office of Science, the U.S. National Science Foundation and the Smithsonian Institution, and by NSERC in Canada. We acknowledge the excellent work of the technical support staff at the Fred Lawrence Whipple Observatory and at the collaborating institutions in the construction and operation of the instrument. The VERITAS Collaboration is grateful to Trevor Weekes for his seminal contributions and leadership in the field of VHE gammaray astrophysics, which made this study possible. 

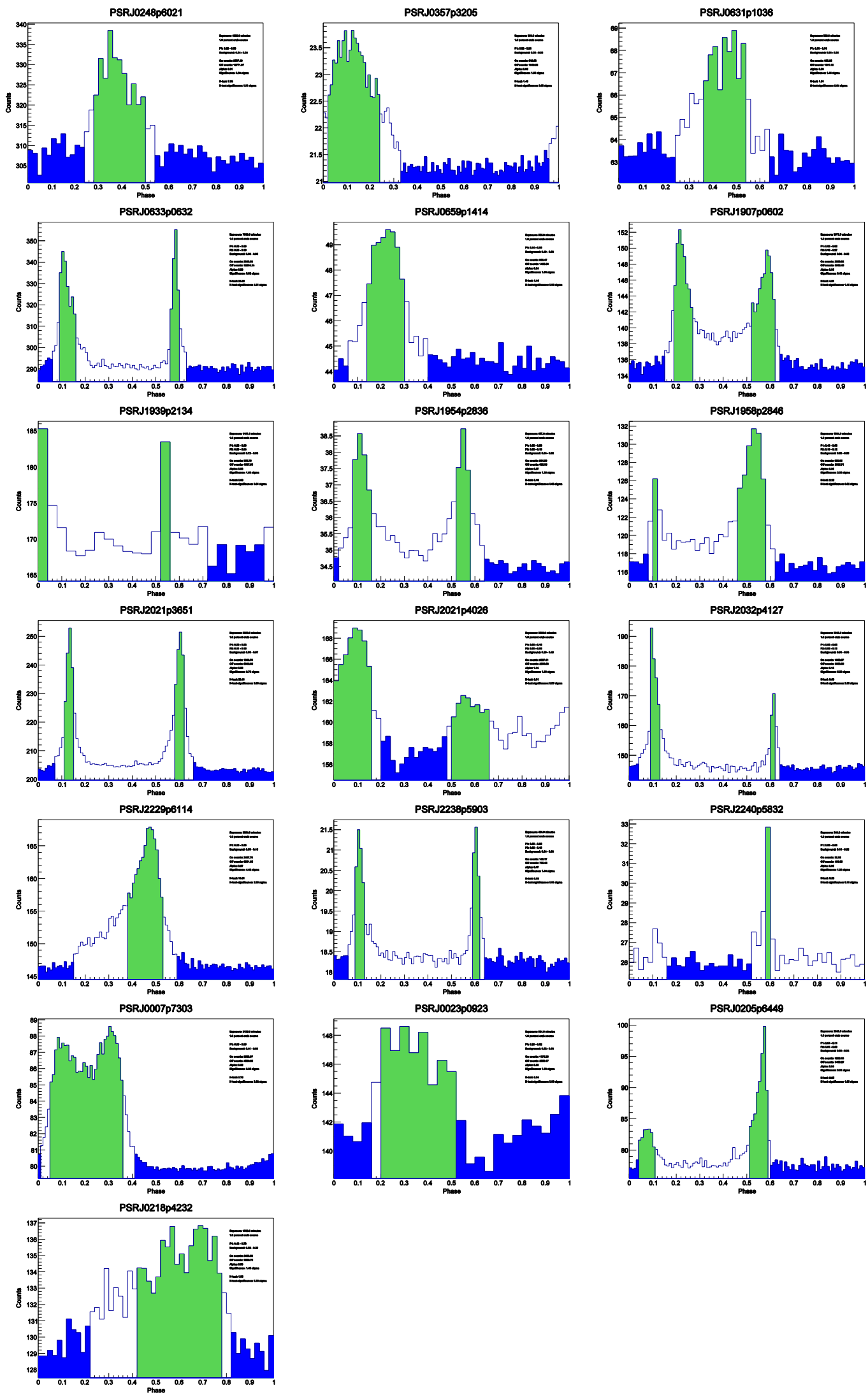

Figure 2. Simulated phaseograms of VERITAS archival pulsars with phase gates defined by the method described. P1 and P2 signal gates are shown in green, background gates are shown in blue. 


\section{References}

[1] Aliu, E., Arlen, T., et al. Detection of Pulsed Gamma Rays Above 100 GeV from the Crab Pulsar, Science, 2011, [arXiv: 1108.3797].

[2] Aleksic, J., Alvarez, E. A., et al., Observations of the Crab pulsar between $25 \mathrm{GeV}$ and $100 \mathrm{GeV}$ with the MAGIC I telescope, Astrophysical Journal, 2011, [arXiv: 1108.5391].

[3] A. A. Abdo et al., The First Fermi Large Area Telescope Catalog Of Gamma-Ray Pulsars, Astrophysical Journal Supplement Series, 2010, [arXiv: 0910.1608].

[4] Thompson D.J., Gamma Ray Astrophysics: the EGRET Results, Reports on Progress in Physics, 2008, [arXiv: 0811.0738].

[5] Romani, R.W., Gamma-Ray Pulsars: Radiation Processes in the Outer Magnetosphere, Astrophysical Journal, 1996.

[6] Kuiper L., COMPTEL Detection of Pulsed Emission from PSR B1509-58 Up to at Least $10 \mathrm{MeV}$, Astrophysical Letters and Communications, 1999, [arXiv: astro-ph/9812405].

[7] Lyutikov, M., Otte, N., \& McCann, A. The Very High Energy Emission From Pulsars: A Case For Inverse Compton Scattering, Astrophysical Journal, 2012, [arXiv: 1108.3824].

[8] Du, Y. J., Qiao, G. J., \& Wang, W., Radio-toTeV Phase-resolved Emission from the Crab Pulsar: The Annular Gap Model, Astrophysical Journal, 2012, [arXiv: 1202.1096].

[9] Aharonian, F. A., Bogovalov, S. V., \& Khangulyan, D., Abrupt Acceleration of a 'Cold' Ultrarelativistic Wind from the Crab Pulsar, Nature, 2012, [arXiv: 1207.0458]

[10] http://www.mpg.de/8287998/velar pulsar/

[11] Leung, G. C. K., Takata, J., Ng, C. W., et al., Fermi-LAT Detection of Pulsed Gamma-Rays above 50 GeV from the Vela Pulsar, The Astrophysical Journal Letters, 2014, [arXiv: 1410.5208].

[12] Aliu, E., Archambault, S., Archer, A., et al., A Search for Pulsation from Geminga Above $100 \mathrm{GeV}$ with VERITAS, Astrophysical Journal, 2015, [arXiv: 1412.4734].

[13] McCann A., A Stacked Analysis of 115 Pulsars Observed by the Fermi-LAT, Astrophysical Journal, 2015, [arXiv: 1412.2422].

[14] Abdo, A. A., Ajello, M., Allafort, A., et al., The Second Fermi Large Area Telescope Catalog of Gamma-ray Pulsars, Astrophysical Journal Supplement, 2013, [arXiv:1305.4385].

[15] The Fermi-LAT Collaboration, Fermi Large Area Telescope Third Source Catalog, Astrophysical Journal Supplement, 2015, [arXiv: 1501.02003]

[16] Bochenck, C. and McCann, A., On the Spectral Shape of Gamma-Ray Pulsars Above the Break Energy, in proceedings of $34^{\text {th }}$ International Cosmic Ray Conference, 2015.

[17] Holder, J., et al., American Institute of Physics 303 Conference Series, 2008.

[18] Berge, D., et al. Background Modelling in Very-High-Energy Gamma-ray Astronomy, Astronomy and Astrophysics, 2007, [arXiv: astro-ph/0610959].

[19] T.P. Li and Y.Q. Ma., Analysis Methods for Results in Gamma-ray Astronomy, Astrophysical Journal, 1983.

[20] Richards, G.T., Recent Pulsar Results from VERITAS on Geminga and the Missing Link Binary Pulsar PSR J1023+0038, in proceedings of 34th International Cosmic Ray Conference, 2015. 